\title{
COMMENTARY
}

\section{AKI patients have worse long-term outcomes, especially in the immediate post-ICU period}

\author{
Eric AJ Hoste*1,2 and Wouter De Corte ${ }^{3}$ \\ See related research by Gammelager et al., http://ccforum.com/content/16/4/R124
}

\begin{abstract}
Acute kidney injury (AKI) is associated with worse outcome in the acute phase of acute illness but also in the chronic phase. In a large Danish study in this issue of Critical Care, 1-year mortality was higher in patients with AKI than in patients without AKI. Mortality was most important during the first 50 days after admission to the intensive care unit (ICU), whereas after 2 months the survival curves of patients with $\mathrm{AKI}$ and those of patients without AKI were similar. The reasons for this observation are not clear, but protracted critical illness and fragility after acute critical illness probably play important roles. Because we see more and more of these patients, they should be the focus of ICU research. Consequently, ICU and post-ICU care for these patients requires focus and a more integrated approach to the specific problems of these survivors of acute critical illness.
\end{abstract}

Acute kidney injury (AKI) occurs in one third to two thirds of patients in the intensive care unit (ICU) [1-7]. The majority of clinical studies in ICU patients have found that AKI was associated with increased in-hospital mortality $[8,9]$. This effect persists after correction for other confounders in multivariate analyses. We can only speculate why even small increases of serum creatinine lead to worse outcomes. Plausible causes for this are volume overload, inflammation of and adverse effects on other organs (so-called 'organ cross-talk'), and inadequate drug dosing [10].

\footnotetext{
*Correspondence: eric.hoste@ugent.be

'Department of Intensive Care Medicine, ICU, 2-K12C, Ghent University Hospital,

De Pintelaan 185, 9000 Gent, Belgium

Full list of author information is available at the end of the article
}

The adverse effects of an episode of AKI may also persist for longer follow-up times. In their center in Florida, Hobson and Bihorac and their colleagues [11,12] found that, in specific ICU cohorts such as those who are recovering from major surgery or cardiac surgery, AKI leads to worse outcomes over a period of years. In a large cohort of hospitalized veterans, mortality in patients who had 90-day survival was higher in patients with AKI, and there was a stepwise increase for increasing AKI severity class [13]. In this issue of Critical Care, Gammelager and colleagues [14] nicely demonstrated similar findings in a large cohort of general ICU patients recruited in a large region in Denmark. The authors identified more than 30,000 patients during a 6-year study period. More than $15 \%$ had AKI at ICU admission. Among patients surviving for 30 days, 1-year mortality rates were 20.5\% for the AKI-Risk group, $23.8 \%$ for the AKI-Injury group, and $23.2 \%$ for the AKI-Failure group in comparison with $10.7 \%$ for the patients without AKI. The strengths of the study are the relatively large sample size, the multicenter setting, and the complete follow-up data. The increased risk for long-term worse outcome persisted in different subgroups, making it even more plausible that the increased mortality observed is not caused by a confounder but indeed is associated with the study variable AKI itself.

How can we explain this increased long-term mortality after an episode of AKI? The data presented in the article by Gammelager and colleagues do not provide an answer for this. We can only speculate on the possible link between an episode of AKI and long-term mortality. One of the most claimed reasons for long-term worse outcomes after AKI is incomplete renal recovery [12,15], which covers a spectrum from patients whose glomerular filtration rate is discrete lower than their kidney function was beforehand to patients who remain dependent on dialysis. Incomplete renal recovery is most likely in patients who had the most severe stage of AKI (that is, AKI treated by renal replacement therapy), and the effects on mortality will be most pronounced in patients who remain dependent on dialysis. Unfortunately, the 
authors could not provide data on renal recovery, such as a serum creatinine or estimated glomerular filtration rate at the time of hospital discharge or, for example, 3- or 6-month follow-up time. However, we question whether incomplete renal recovery did play an important role in their findings. In the study, a total of 987 patients with AKI were treated with renal replacement therapy. When ICU mortality of these patients is comparable to that of patients in other Western countries, approximately $50 \%$, or 493, of the former will have died. Of the survivors, $10 \%$ to $25 \%$ (or 50 to 123 ) will remain dialysis-dependent. Here, as many as 642 patients died after day 30 . So nondialysis-dependent incomplete renal recovery would account for $80 \%$ of the additional deaths. Although every nephrologist will confirm that decreased kidney function has an impact on long-term outcomes, the implications are measurable only when observed over a period of several years. In other words, incomplete renal recovery may explain only in part the impressive mortality between day 30 and 1 year as observed in this study.

Careful analysis of the cumulative mortality curves reveals that there was a biphasic pattern: a steep increase in mortality from ICU admission until day 50 and a relatively flat curve afterwards. Importantly, the second half of the curve for patients with AKI is very similar to that for patients without AKI. As $25 \%$ of patients with AKI were still hospitalized after day 30 , it is very likely that the steep curve still represents in-hospital mortality. In other words, patients who survived the immediate 1-month period after AKI died in the second month after AKI diagnosis - a period still linked to the original disease that lay at the origin of AKI.

This is an observation that makes sense. Modern-day intensivists are challenged by the care for patients who survived a first episode of critical illness and stay in the unit and hospital for several weeks. Why patients with AKI do still worse in this period is not clear and should be evaluated in future studies. Apart from this, several lessons can be learned from these observations. First, in modern-day ICU care, we should aim for endpoints that are more relevant, such as 60- or 90-day survival instead of the classic 30-day survival. Second, we are challenged by care for initial ICU survivors, who remain fragile and in moderate organ dysfunction for longer periods of time. The challenges that we face for these patients are not fully understood. In the ICU, we probably need to develop new therapies that are in contrast to the immediate 'point and shoot' approach of the old-style intensivists. When these patients are discharged to a step-down ward, they should be cared for by a team that is specialized in their care. As a consequence, we need to look beyond simple ICU mortality. There is a growing consensus that other issues of long-term outcome, such as quality of life, are worth studying [16].
In conclusion, we should increase our knowledge of these initial ICU survivors, try to elucidate why patients who had an episode of AKI do particularly worse, and develop integrated care with a focus on immediate, but also long-term, outcomes.

\section{Abbreviations}

AKI, acute kidney injury; ICU, intensive care unit.

\section{Competing interests}

The authors declare that they have no competing interests.

\section{Author details}

'Department of Intensive Care Medicine, ICU, 2-K12C, Ghent University Hospital, De Pintelaan 185, 9000 Gent, Belgium. ${ }^{2}$ Research Foundation, Flanders, Egmontstraat 5, 1000 Brussels, Belgium. ${ }^{3}$ Department of Anaesthesia and Intensive Care Medicine, AZ Groeninge Hospital, Pres. Kennedylaan 4, 8500 Kortrijk, Belgium.

\section{Published: 27 August 2012}

\section{References}

1. Hoste EA, Clermont G, Kersten A, Venkataraman R, Angus DC, De Bacquer D, Kellum JA: RIFLE criteria for acute kidney injury are associated with hospital mortality in critically ill patients: a cohort analysis. Crit Care 2006, 10:R73.

2. Ostermann M, Chang RW: Acute kidney injury in the intensive care unit according to RIFLE. Crit Care Med 2007, 35:1837-1843.

3. Ostermann M, Chang R: Correlation between the AKI classification and outcome. Crit Care 2008, 12:R144.

4. Bagshaw SM, George C, Dinu I, Bellomo R: A multi-centre evaluation of the RIFLE criteria for early acute kidney injury in critically ill patients. Nephrol Dial Transplant 2008, 23:1203-1210.

5. Bagshaw SM, George C, Bellomo R: A comparison of the RIFLE and AKIN criteria for acute kidney injury in critically ill patients. Nephrol Dial Transplant 2008, 23:1569-1574.

6. Joannidis M, Metnitz B, Bauer P, Schusterschitz N, Moreno R, Druml W, Metnitz PG: Acute kidney injury in critically ill patients classified by AKIN versus RIFLE using the SAPS 3 database. Intensive Care Med 2009, 35:1692-1702.

7. Mandelbaum T, Scott DJ, Lee J, Mark RG, Malhotra A, Waikar SS, Howell MD, Talmor D: Outcome of critically ill patients with acute kidney injury using the Acute Kidney Injury Network criteria. Crit Care Med 2011, 39:2659-2664

8. Hoste EAJ, Schurgers M: Epidemiology of AKI: How big is the problem? Crit Care Med 2008, 36:S1-4.

9. Ricci Z, Cruz D, Ronco C: The RIFLE criteria and mortality in acute kidney injury: a systematic review. Kidney Int 2008, 73:538-546.

10. Hoste EA, De Corte W: Clinical consequences of acute kidney injury. Contrib Nephrol 2011, 174:56-64.

11. Bihorac A, Yavas S, Subbiah S, Hobson CE, Schold JD, Gabrielli A, Layon AJ, Segal MS: Long-term risk of mortality and acute kidney injury during hospitalization after major surgery. Ann Surg 2009, 249:851-858.

12. Hobson CE, Yavas S, Segal MS, Schold JD, Tribble CG, Layon AJ, Bihorac A: Acute kidney injury is associated with increased long-term mortality after cardiothoracic surgery. Circulation 2009, 119:2444-2453.

13. Lafrance JP, Miller DR: Acute kidney injury associates with increased longterm mortality. J Am Soc Nephrol 2010, 21:345-352.

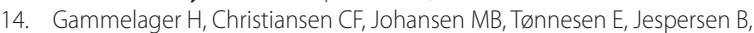
Sørensen HT: One-year mortality among Danish intensive care patients with acute kidney injury: a cohort study. Crit Care 2012, 16:R124.

15. Schiff H, Fischer R: Five-year outcomes of severe acute kidney injury requiring renal replacement therapy. Nephrol Dial Transplant 2008, 23:2235-2241

16. Oeyen SG, Vandijck DM, Benoit DD, Annemans L, Decruyenaere JM: Quality of life after intensive care: a systematic review of the literature. Crit Care Med 2010, 38:2386-2400.

\section{doi:10.1186/cc11470}

Cite this article as: Hoste EAJ, De Corte W: AKI patients have worse longterm outcomes, especially in the immediate post-ICU period. Critical Care 2012, 16:148, 